\title{
A SIMPLIFIED FINITE ELEMENT FORMULATION OF SHALLOW FOUNDA- TIONS FOR CIVIL ENGINEERING STRUCTURES SEATED ON SOFT CLAY SOIL
}

\author{
M. Moreno ${ }^{1}$, N. Dominguez ${ }^{1}$
}

${ }^{1}$ Department of Postgraduates and Research (SEPI ESIA UZ), National Polytechnic Institute of Mexico, Av. Miguel Bernard s/n, Col. Lindavista Zacatenco, 07300, Mexico D.F. Mexico (miguelmoreno77@gmail.com, ndominguez@ipn.mx )

\begin{abstract}
The estimation of the structural response of civil engineering structures as they are subjected to different combination of loadings is currently done by the use of numerical models, and its effectiveness depends on how boundary conditions are included into the model. In the case of constructions seated on clay soils, a numerical difficulty resides in how to model the soil-foundation interaction as well as the non linear material behavior of soil. In this work a practical engineering solution is presented in which a set of some simplified foundation elements is integrated into the conventional analysis of a building. A simplified numerical strategy of four steps is proposed in order to include the soil-foundation interaction into the calculations of a global structure. Regarding to the soil-foundation interaction, two superelements called "Isolated Foundation Element (IFE)" and "Continuous Foundation Element (CFE)" are numerically formulated, being able to take into account the non linear material behavior of a stratified soil -in particular, long term deformability -, the influence of the soil region in the vicinity of the embedded foundation and their effects in the whole structural system. Both formulations include different criteria for evaluating soil stress distribution (Boussinesq, Westergard, Fhröhlich) as well as the evaluation of the volumetric deformation for a stratified soil (Zeevaert semi-empirical criteria). In order to evaluate the robustness of the proposed strategy, some foundation cases are analyzed in the finite element codes ANSYS and GeoStudio, as well as using the IFE/CFE elements: the comparison of the numerical results shows a good performance of the proposal, with a reduced number of calculations and less complexity.
\end{abstract}

Keywords: Shallow foundations, super-finite elements, stratified soft soil, soil-structure interaction.

\section{INTRODUCTION}

Structural analysis and design are one of the most important activities in civil engineering, due to the fact that the final dimensions and the characteristics of all structural elements depend on the quality of the numerical results obtained according to the selected analysis method and the set of adopted hypotheses. In the case of buildings settled on soft soil, the 
integration of the Soil-Structure Interaction phenomenon (SSI) into the analysis affects directly the response of the whole structural system and consequently the distribution of internal forces and displacements. Taking into account that soil might show a strong non linear material behavior due to the inherent uncertainties, the complexity of the problem increases dramatically in terms of numerical cost and difficulty. In face of this situation, one possible solution is to simulate the whole SSI system using a finite element code in which the slab foundation might be modeled with shell elements, while the soil mass could be modeled with either one-dimensional springs or 3D brick elements. Evidently, this becomes very complicated even for just one slab foundation...

Because of this, typical structural engineers prefer to deal with simplified, fast and conservative static methods based on linear elastic material behavior, instead of using powerful finite element programs: the most risky inconvenient of these practices is not only to obtain an expensive and not-optimized final design, but also this foundation design might be apparently conservative, because the real response of the structure depends directly on the boundary conditions which could be mischaracterized (e.g., the fact of introducing a fixed support under each column without taking care of a strong non linear behavior of multilayered clay soil).

The aim of this work is to propose a simplified numerical strategy that relies on the formulation of two super-elements called respectively the "Isolated Foundation Element (IFE)" and the "Continuous Foundation Element (CFE)", which are able to take into account the non linear properties of a stratified soil -in particular, long term deformability -, the influence of the soil region in the vicinity of the embedded slab and their effects in the whole structural system. In order to consider the effect of soil settlement in the global response, the model includes different soil stress distribution's criteria (Boussinesq, Westergaard and Fhrohlich methods [2][4]) as well as the phenomenological model proposed by Zeevaert [1] to evaluate the volumetric deformation for a stratified soil. The aim of this proposal is to improve the design practices widely adopted for most of all structural engineers, by the contribution of dedicated finite elements that can be easily adapted into any standard finite element code without affecting its internal structure.

\section{DESCRIPTION OF THE NUMERICAL PROPOSAL}

\subsection{The numerical strategy for analysis}

The proposed numerical strategy to analyze a simple foundation-clay soil system follows the next steps:

1. Definition of the influence regions: once that foundation layout has been well defined, the total area under foundation is divided in small areas that we call "influence region", whose boundaries might be beyond construction limits, if some neighboring loads affecting the soil will be considered into the analysis (see figure 1). 


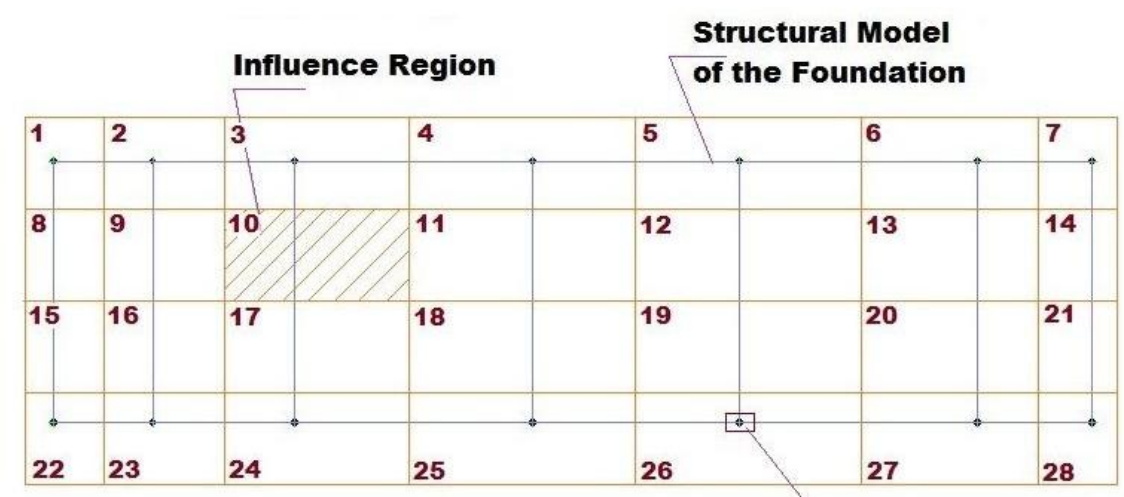

Isolated Foundation Element IFE

Figure1. Definition of the influence region

2. Analysis of the local settlements: for each influence region, a study of local settlements evaluated on the ground level is done, based on the experimental semiempirical criterion developed by Zeevaert [1]. This criterion takes into account a realistic stress-strain-time curve of each clay-soil layer registered on the field and an experimental parameter "alpha" for each stratum, and applying some semi-empirical equations, evaluates the vertical displacement of each layer. Placing a unitary distributed vertical pressure on the local influence region, an influence region matrix can be calculated and assembled, as it is shown in figure 2 .

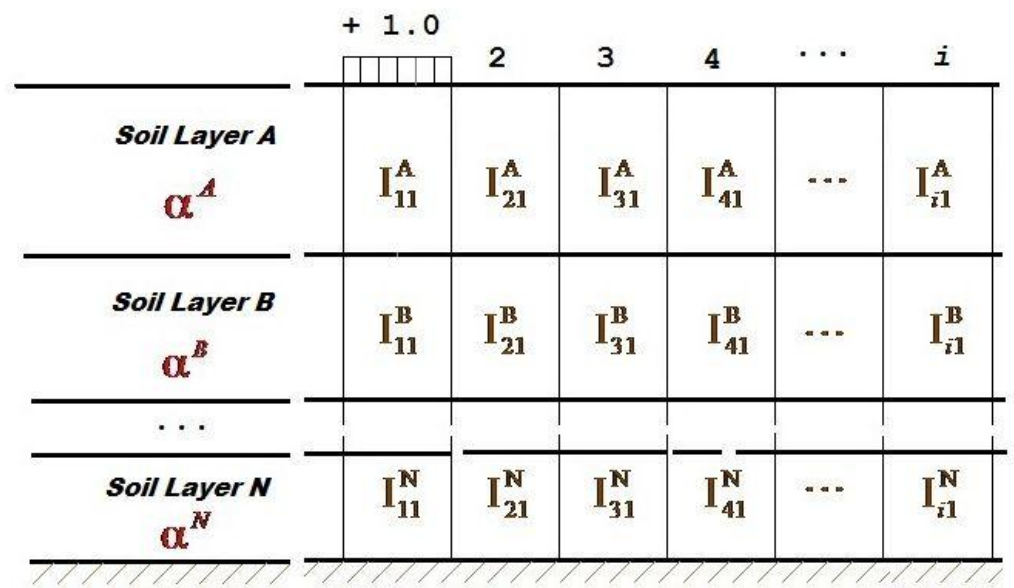

Figure2. Soil layers and influence regions in the vertical direction

3. Selection of the built-in foundation element: A foundation mesh based in classical finite elements is built, including only the strap-beams and either the IFE elements or the CFE elements, placed in their respective locations (see figure 3). 


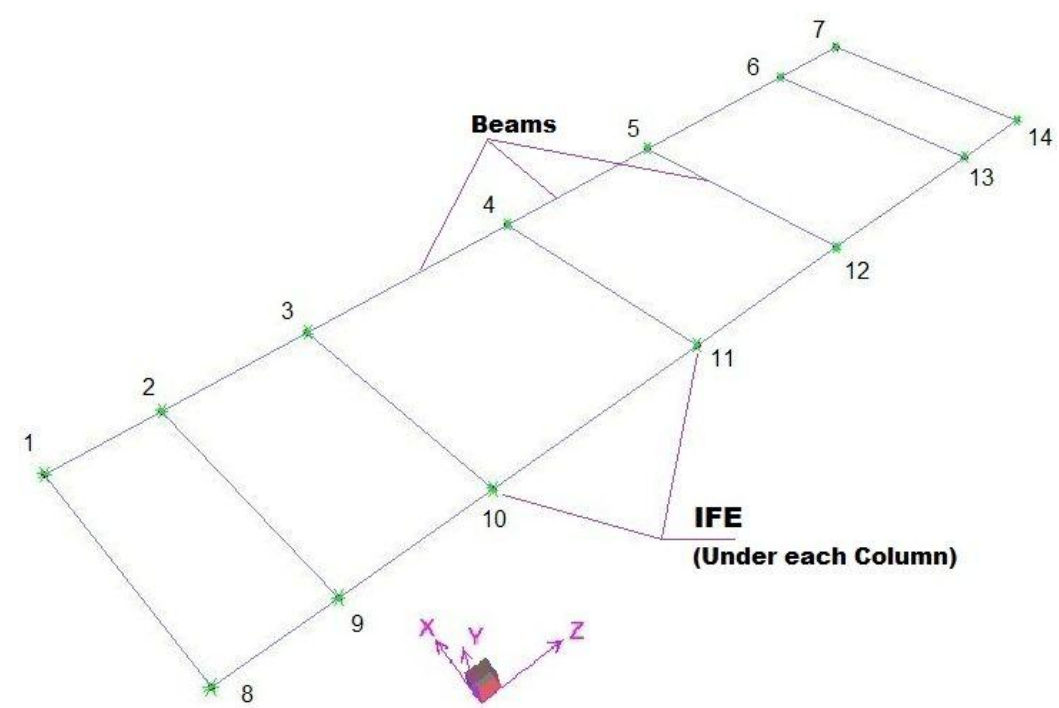

Figure3. Simplified model of the strap-beam - IFE/CFE system

4. Application of the iterative analysis: The procedure of the iterative numerical analysis is as follows:

a) A first elastic analysis cycle is done only for the foundation system, in which the same initial value of the modulus of subgrade reaction is assigned for all IFE/CFE elements.

b) The obtained reactions are transferred to each influence region as loadings, including the other overloads produced by neighboring constructions, roads, retaining walls, etc. (see figure 4).

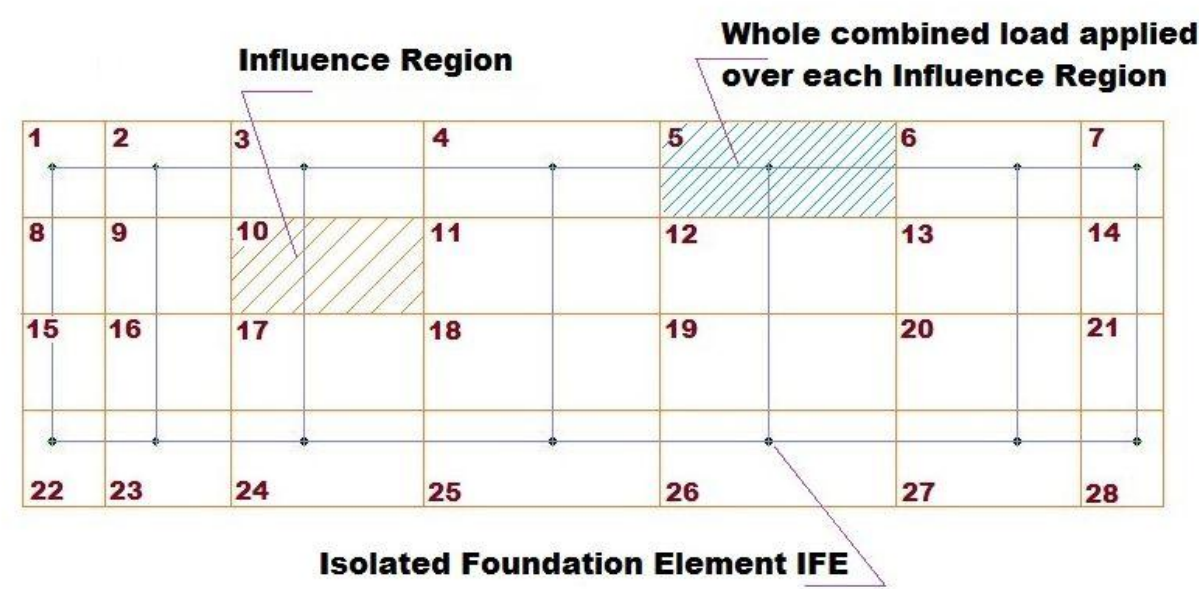

Figure4. Combined loads applied over influence regions

c) The superficial ground settlements are calculated by solving the equation system defined by the influence region matrix and the overloads vector, according to the equation (1):

$$
\left[\delta_{i}\right]=\left[\bar{\delta}_{j i}\right]^{T} \cdot\left[q_{i}\right]
$$


d) For each iterative cycle " $k$ ", an equivalent modulus of subgrade reaction is calculated for each " $i$ " influence region, and this value is replaced in the corresponding IFE/CFE element, according to the equation (2):

$$
\left[k_{0_{i}}\right]_{k}=\left(\frac{P_{i}}{\delta_{i}}\right)_{k}
$$

By the way, the elastic stiffness coefficients of each IFE/CFE element for the iterative cycle $k$, are determined by the set of equations 3 (In the case of CFE elements, equations (b) and (c) are not taken into account):

$$
\begin{aligned}
K_{y} & =k_{0} \cdot A \cdot B \\
K_{\phi x} & =k_{0} \cdot \frac{A \cdot B^{3}}{12} \\
K_{\phi z} & =k_{0} \cdot \frac{A^{3} \cdot B}{12}
\end{aligned}
$$

e) A new structural analysis of the foundation is done for the next iterative cycle.

f) The steps (b) and (e) are repeated until the convergence is satisfied, or as soon as a predefined number of iterative cycles, is reached. In this case, the convergence criterion is verified when the variation of the modulus of subgrade reaction for each influence zone is less than an accepted error value, according to the equation (4).

$$
\varepsilon=\left|\frac{\left[k_{0_{i}}\right]_{k}-\left[k_{0_{i}}\right]_{k-1}}{\left[k_{0_{i}}\right]_{k}}\right| .100 \leq \text { Tol } .
$$

\subsection{Theoretical formulation of the IFE/CFE elements}

Here, we present the numerical formulation of the Isolated Foundation Element (IFE) as well as the Continuous Foundation Element (CFE). Three degrees of freedom are associated to the IFE, being: (a) translational vertical displacement on the Y-axis; (b) rotational motion around X-axis; and (c) rotational motion around $\mathrm{Z}$-axis. In the case of the CFE element, the stiffness matrix is enriched with the contribution of the support. In the other hand, the required parameters to define any IFE/CFE element are:

- Geometrical parameters (horizontal length A and B, and thickness)

- Global modulus of subgrade reaction

- Overloading 
Locally, the stiffness coefficients of the IFE/CFE foundations are calculated with the set of equations (3) mentioned before. Once the IFE/CFE elementary stiffness matrix is constructed, it must be reassembled from the local to the global space through the use of a classical transformation matrix. In Figures $5 \mathrm{a}$ and $5 \mathrm{~b}$, the calculations of the rotational and translational stiffness for the IFE element are presented respectively. In Figure 6 it is possible to verify the construction of the stiffness matrix for the CFE.

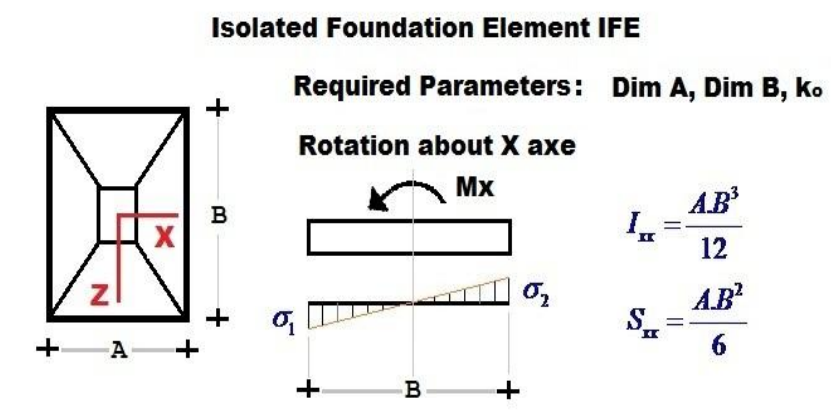

$$
\begin{aligned}
& \text { Equilibrium Equation: } \quad M=K_{R} \theta \\
& \text { Stress : } \sigma_{1}=M x / S_{\text {Ix }}=6 M x / A B^{2} \therefore M x=A B^{2} / 6 . \sigma_{1} \\
& \begin{array}{c}
\text { Translational and } \\
\text { Rotational : }: d=\sigma / k_{0}
\end{array} \text { y } \quad \theta_{\mathrm{x}}=2 \cdot d / B \\
& \therefore \sigma=d k_{0}=\frac{1}{2} \theta_{x} B k_{0}
\end{aligned}
$$

Replacing in the Equilibrium Equation :

$$
M x=\frac{A B^{2}}{6} \cdot \frac{\theta_{x} B}{2} k_{0}=\underbrace{\frac{A B^{3}}{12} k_{0} \cdot \theta_{x} \quad \therefore K_{j=}=k_{0}-\frac{A B^{3}}{12}}_{\text {Stiffness }}
$$

Figure5a. Formulation of the IFE rotational stiffness

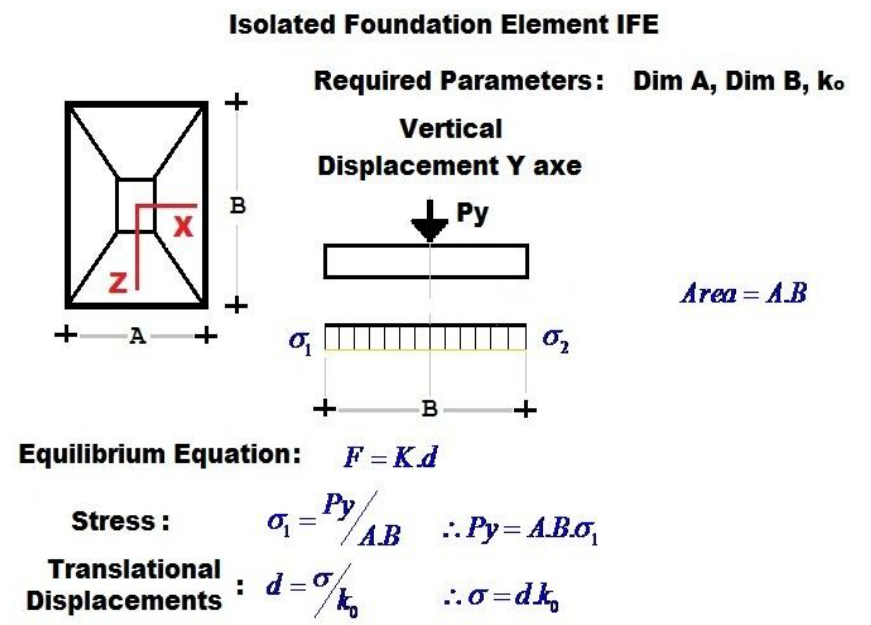

Replacing in the Equilibrium Equation :

$$
P y=\underbrace{A \cdot B k_{0} d}_{\text {Stiffness }} \quad K_{y}=k_{0}-A B
$$

Figure 5b. Formulation of the IFE vertical displacement stiffness 
Continuous Foundation Element CFE

Required Parameters : L, A, B1, B2, d, $k_{\circ}$, w

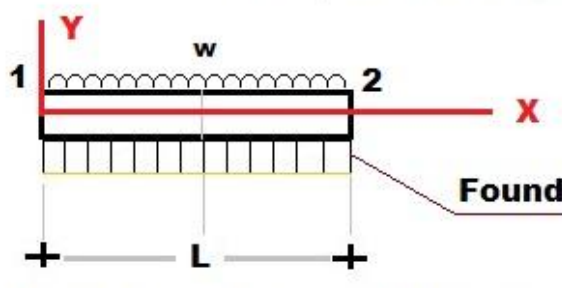

Equilibrium Differential Equation

$E I \frac{\partial^{4} y}{\partial x^{4}}+k y=w$

Active Node Degree

$\left[\begin{array}{l}\theta_{x}-\text { Rotation X Axis } \\ \delta_{y}-\text { Vertical Displ Y } \\ \theta_{z}-\text { Rotation Z Axis }\end{array}\right]$

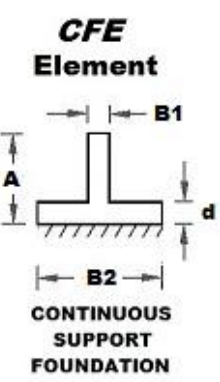

of Freedom Consider

CFE Local Element Stiffness Matrix

$$
K=K \text { (elastic beam) }+K \text { (support contribution) }
$$

$$
\begin{aligned}
& \mathbf{K}=\left[\begin{array}{cccccc}
\frac{G J_{m}}{L} & 0 & 0 & -\frac{G J_{m}}{L} & 0 & 0 \\
0 & \frac{12 E I}{\left(1+\emptyset_{y}\right) L^{3}} & \frac{6 E I}{\left(1+\emptyset_{y}\right) L^{2}} & 0 & -\frac{12 E I}{\left(1+\emptyset_{y}\right) L^{3}} & \frac{6 E I}{\left(1+\emptyset_{y}\right) L^{2}} \\
0 & \frac{6 E I}{\left(1+\emptyset_{y}\right) L^{2}} & \frac{\left(4+\emptyset_{y}\right) E I}{\left(1+\emptyset_{y}\right) L} & 0 & -\frac{6 E I}{\left(1+\emptyset_{y}\right) L^{2}} & \frac{\left(2-\emptyset_{y}\right) E I}{\left(1+\emptyset_{y}\right) L} \\
-\frac{G J_{m}}{L} & 0 & 0 & \frac{G J_{m}}{L} & 0 & 0 \\
0 & -\frac{12 E I}{\left(1+\emptyset_{y}\right) L^{3}} & -\frac{6 E I}{\left(1+\emptyset_{y}\right) L^{2}} & 0 & \frac{12 E I}{\left(1+\emptyset_{y}\right) L^{3}} & -\frac{6 E I}{\left(1+\emptyset_{y}\right) L^{2}} \\
0 & \frac{6 E I}{\left(1+\emptyset_{y}\right) L^{2}} & \frac{\left(2-\emptyset_{y}\right) E I}{\left(1+\emptyset_{y}\right) L} & 0 & -\frac{6 E I}{\left(1+\emptyset_{y}\right) L^{2}} & \frac{\left(4+\emptyset_{y}\right) E I}{\left(1+\emptyset_{y}\right) L}
\end{array}\right] \\
&+\left.\begin{array}{cccccc}
0 & 0 & 0 & 0 & 0 & 0 \\
0 & \frac{13 k L}{35} & \frac{11 k L^{2}}{210} & 0 & \frac{9 k L}{70} & -\frac{13 k L^{2}}{420} \\
0 & \frac{11 k L^{2}}{210} & \frac{k L^{3}}{105} & 0 & \frac{13 k L^{2}}{420} & -\frac{k L^{3}}{140} \\
0 & 0 & 0 & 0 & 0 & 0 \\
0 & \frac{9 k L}{70} & \frac{13 k L^{2}}{420} & 0 & \frac{13 k L}{35} & -\frac{11 k L^{2}}{210} \\
0 & -\frac{13 k L^{2}}{420} & -\frac{k L^{3}}{140} & 0 & -\frac{11 k L^{2}}{210} & \frac{k L^{3}}{105}
\end{array}\right]
\end{aligned}
$$

where : $\emptyset_{y}=\frac{12 f_{y} E I}{A G L^{2}} ; k=B 2 . k_{o} ; f_{y}$ shear form factor ; $J_{m}$ torsional constant

Figure6. Formulation of the CFE stiffness 


\section{NUMERICAL IMPLEMENTATION ON A FINITE ELEMENT CONTEXT}

The precedent numerical strategy has been implemented in a typical 3D FE code called "FINITO", developed by the first author in the National Polytechnic Institute of Mexico, without affecting the internal structure of the code. In which concerns to the local analysis of the IFE/CFE elements, a classical Newton-Raphson algorithm was adopted as iterative method, having a general initial value of the modulus of subgrade reaction that will be corrected in each iterative cycle. The local iterative algorithm for the IFE element is fully described in figure 7 (CFE element's algorithm can be deduced from it) while the flow diagram showing the global procedure of analysis is presented in figure 8 .

\section{Finite Element IFE}

\section{Parameter's Definition}

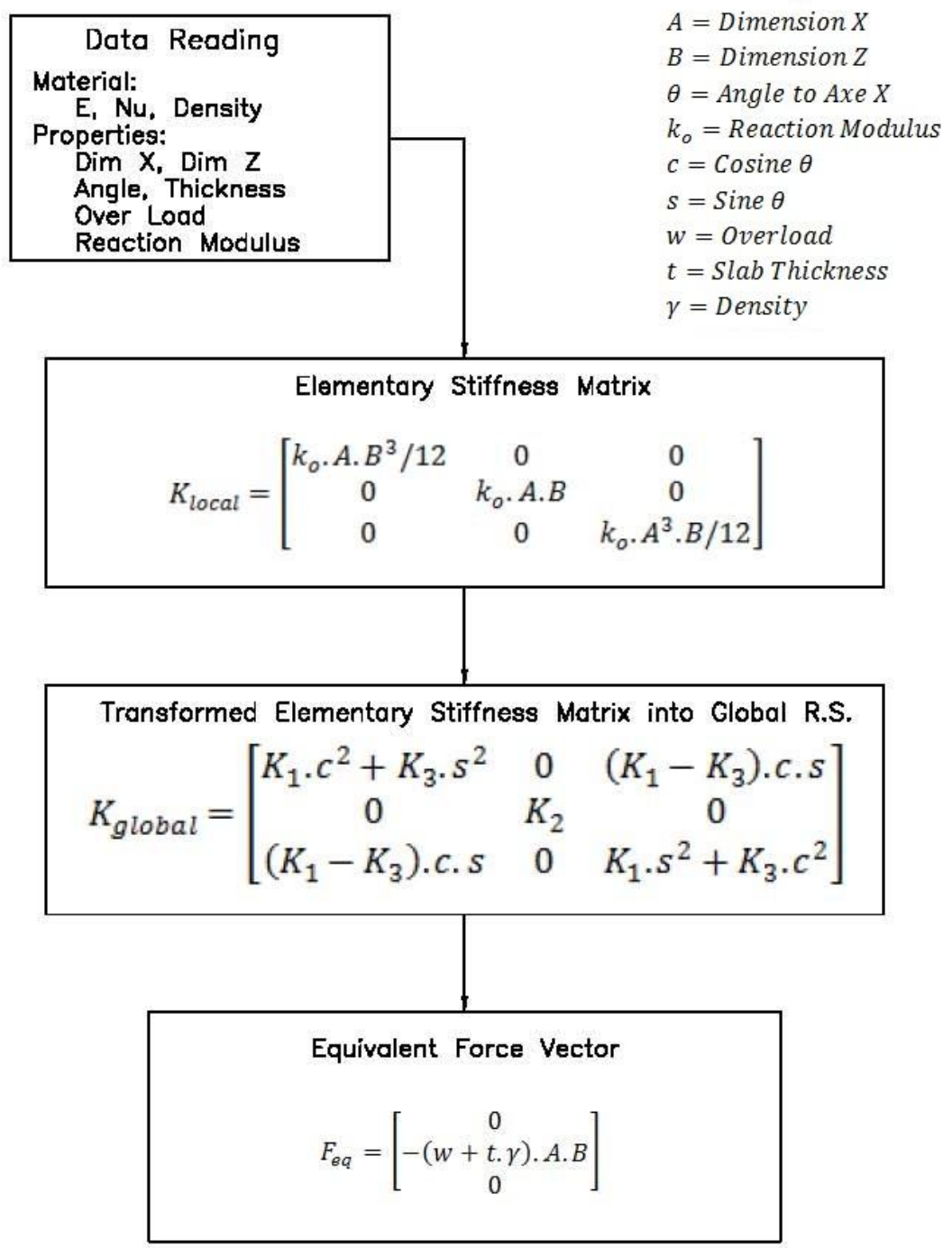

Figure7. Local flow diagram of the IFE's internal calculation 


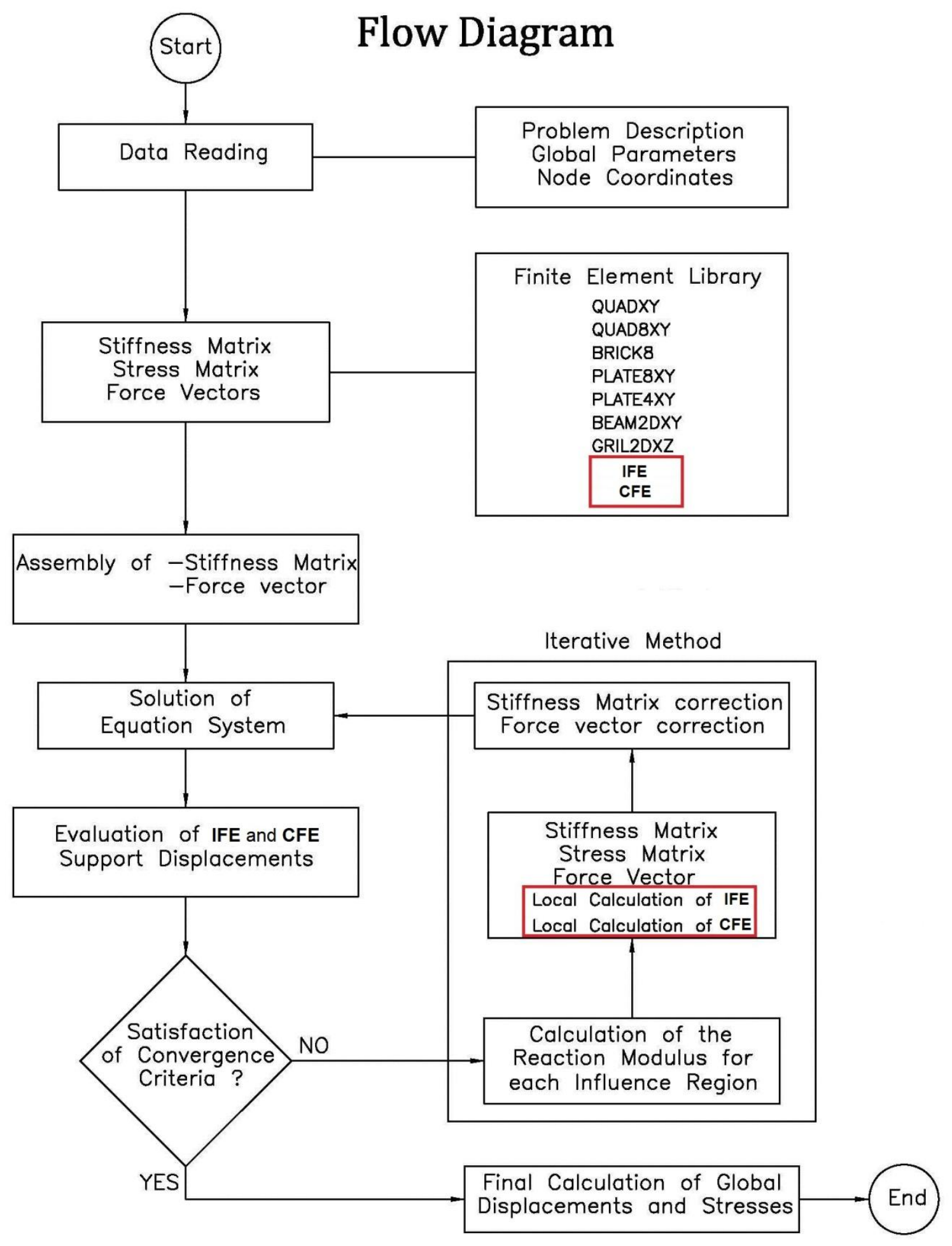

Figure8. Global flow diagram of the proposed strategy 


\section{ANALYSIS OF A STANDARD PROBLEM AND COMPARISON OF RESULTS}

In order to evaluate this proposal, a practical example is analyzed with different boundary conditions using several numerical methods. It consists in a $2 \mathrm{D}$ frame composed by three columns and two beams with different non symmetric static loads. In all cases, the soil is composed by three layers of clay with different characteristics, as it is shown in figure 9. In the first case, the frame is analyzed considering the support-nodes as fully restrained (see figure 10). In the second case (figure 11), the soil is modeled using the classical Winkler springs. In the third case, the "Isolated Foundation Element" proposed in this work are placed under each column (see figure 12). In the fourth case, a continuous slab is simulated under the structure, linking the three columns of the frame with beams that are combined with springs underneath (see figure 13). Finally in the last case, the proposed "Continuous Foundation Element" is placed directly under the columns and integrated into the calculations (see figure 14). The results (displacements and reactions) of each case -except for case 5-are presented in the respective Tables 1, 2, 3 and 4. In case 5, the loading of the structure is spread over the influence region, and thus instead of nodal reactions, a value of pressure is deduced.

By comparing these results, it is clearly observed that response depends on how the frame is supported: looking at node five, which is the most loaded point, there is no displacement for case 1, while in case 2 (with Winkler springs) the vertical displacement obtained has a value of $0.657 \mathrm{~cm}$; for case 3 in which IFE elements are incorporated, the value of the vertical displacement is $4.959 \mathrm{~cm}$. This could be explained by two facts: (a) the combined effect of the deformation of each layer of soil increases the vertical displacement; and (b) the interdependency between the IFE elements, which does not exist in the case of the Winkler springs, makes a better distribution of the efforts. In case 4 , in which a continuous slab is supposed to be simulated, the value of the vertical displacement is about $0.609 \mathrm{~cm}$, very close to the value obtained in case 2 . This could be explained by the impossibility of transmission of efforts between the soil's springs, leaving the strap-beams as the only way of transferring forces on the structural system. In the other hand, in case 5 in which CFE elements are included to simulate the continuous slab, a value of $4.741 \mathrm{~cm}$ is calculated under node five, but the maximal value of vertical displacement is obtained in node $6(8.94 \mathrm{~cm})$ : this is a very interesting result, because that means that the deformability of the layered soil produces a redistribution of efforts under the nodes affecting the stiffness of the soil, and modifying the structural response of the system.

In which concerns to the design of the structural elements of the frame, it is necessary to evaluate the distribution and magnitude of the internal mechanical forces obtained from each analysis. So, the question is: what occurs in the internal behavior of the structural elements? In figure 15, a comparison of the shear forces and flexural moments on beams is shown for cases 1, 2 and 3. In the left beam, the differences between cases 1 and 2 are irrelevant, but in case 3 in which IFE elements are included, there is a non neglected redistribution of internal forces: the maximal absolute value of shear force decreases in approximately $15 \%$ (from $15 \mathrm{~T}$ to $13 \mathrm{~T}$ ) as well as the maximal negative value of flexural moment which diminishes from 
1500 T.m to about 800 T.m, while the maximal positive value of flexural moment increases from 650 T.m to about 900 T.m. In the right beam, a similar situation occurs: between cases 1 and 2 , there is no relevant difference, while case 3 shows a redistribution of shear forces and flexural moments. Concerning to internal shear force, it increases its maximal absolute value from $20 \mathrm{~T}$ to about $25 \mathrm{~T}$, while the maximal negative value of flexural moment increases from 1000 T.m to 1500 T.m; concerning to the positive flexural moment, most of the values increase along the beam, modifying the envelope, which can affect the design of the reinforcement of the beam (either in concrete or steel).

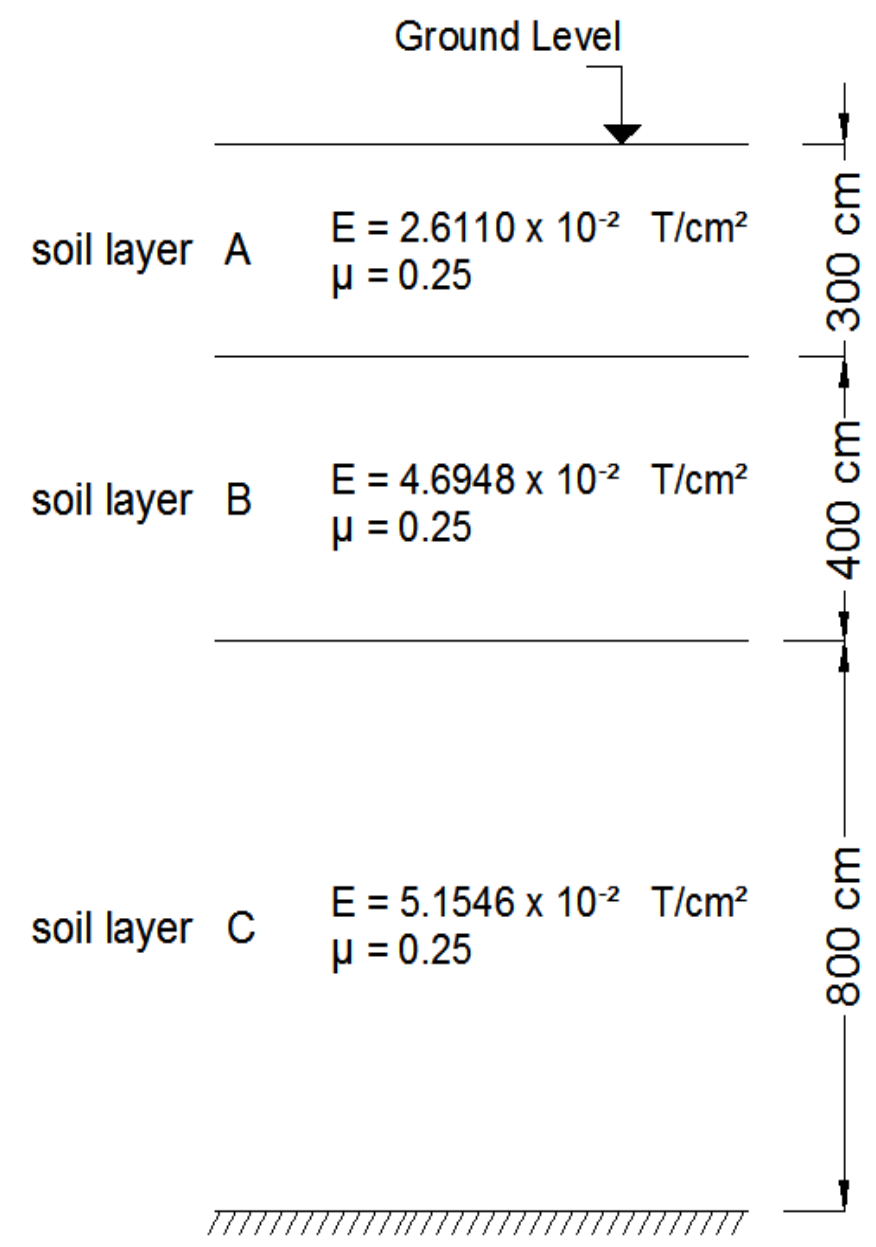

Figure9. Section of the layered soil supporting the structure of the example 


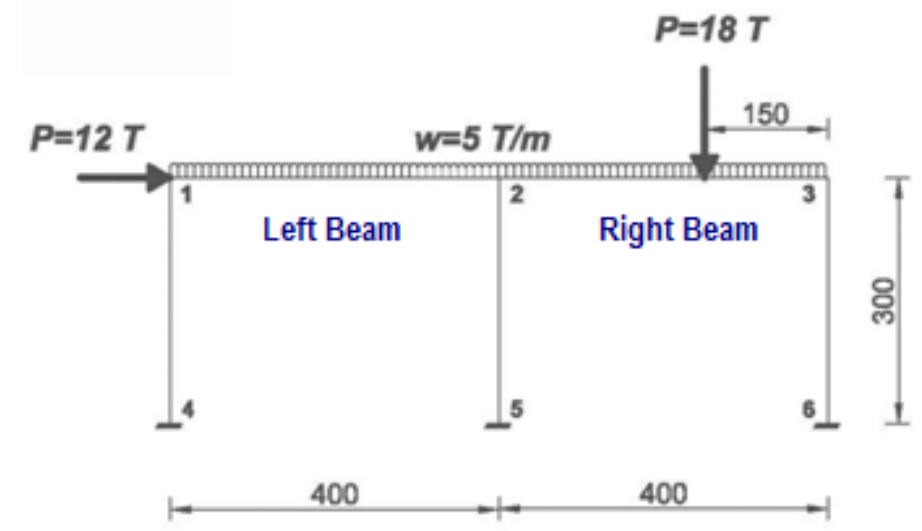

Figure10. 2D frame supported on fixed nodes (case 1)

Table1. Displacements and reactions calculated in case 1

\begin{tabular}{|c|c|c|c|c|c|c|c|}
\hline \multirow[b]{3}{*}{ Node } & \multicolumn{3}{|c|}{ Displacements } & & \multicolumn{3}{|c|}{ Reactions } \\
\hline & $d x$ & dy & Rot $\theta$ & & $\mathrm{Rx}$ & Ry & Mom Z \\
\hline & $(\mathrm{cm})$ & $(\mathrm{cm})$ & (rad) & Node & $(\mathrm{T})$ & $(\mathrm{T})$ & $(\mathrm{T}-\mathrm{cm})$ \\
\hline 1 & 1.320 & -0.011 & -0.00270 & & & & \\
\hline 2 & 1.303 & -0.070 & -0.00210 & 4 & -2.929 & 5.016 & 504.230 \\
\hline 3 & 1.293 & -0.045 & 0.00368 & 5 & -3.166 & 32.286 & 525.249 \\
\hline 4 & 0.000 & 0.000 & 0.00000 & 6 & -5.905 & 20.699 & 797.502 \\
\hline 5 & 0.000 & 0.000 & 0.00000 & & & & \\
\hline 6 & 0.000 & 0.000 & 0.00000 & & & & \\
\hline
\end{tabular}

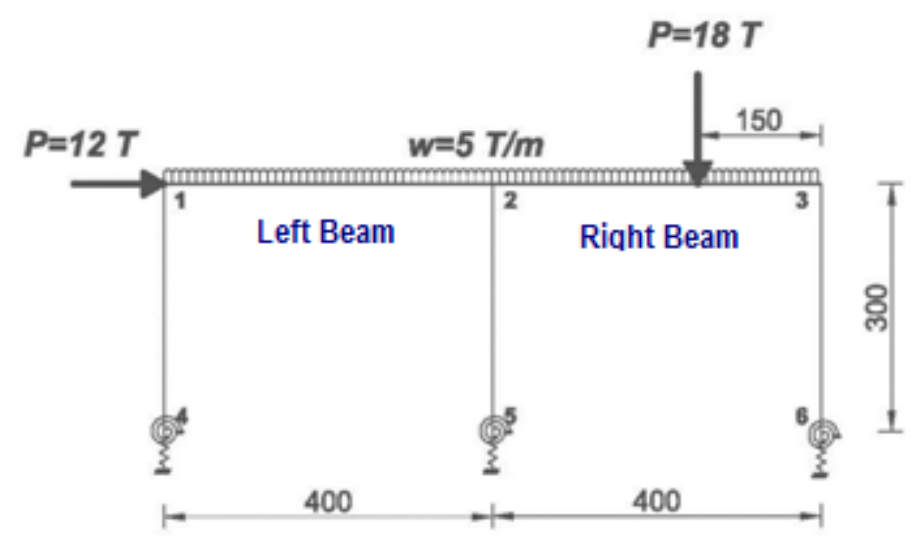

Figure 11. 2D frame supported on pseudo-soil springs (case 2)

Table2. Displacements and reactions calculated in case 2

\begin{tabular}{|c|c|c|c|}
\hline & \multicolumn{3}{|c|}{ Displacements } \\
\hline & $d x$ & dy & $\operatorname{Rot} \theta$ \\
\hline Node & $(\mathrm{cm})$ & $(\mathrm{cm})$ & (rad) \\
\hline 1 & 2.304 & -0.172 & -0.00426 \\
\hline 2 & 2.288 & -0.725 & -0.00306 \\
\hline 3 & 2.278 & -0.747 & 0.00366 \\
\hline 4 & 0.000 & -0.161 & -0.00558 \\
\hline 5 & 0.000 & -0.657 & -0.00372 \\
\hline 6 & 0.000 & -0.701 & -0.00786 \\
\hline
\end{tabular}

\begin{tabular}{|c|c|r|r|}
\hline & \multicolumn{3}{|c|}{ Reactions } \\
\hline & Rx & \multicolumn{1}{c|}{ Ry } & Mom Z \\
\hline Node & \multicolumn{1}{|c|}{$(\mathrm{T})$} & $(\mathrm{T})$ & $(\mathrm{T}-\mathrm{cm})$ \\
\hline & & & \\
\hline 4 & -2.652 & 4.959 & 365.968 \\
\hline 5 & -4.070 & 31.521 & 594.693 \\
\hline 6 & -5.278 & 21.520 & 515.048 \\
\hline
\end{tabular}




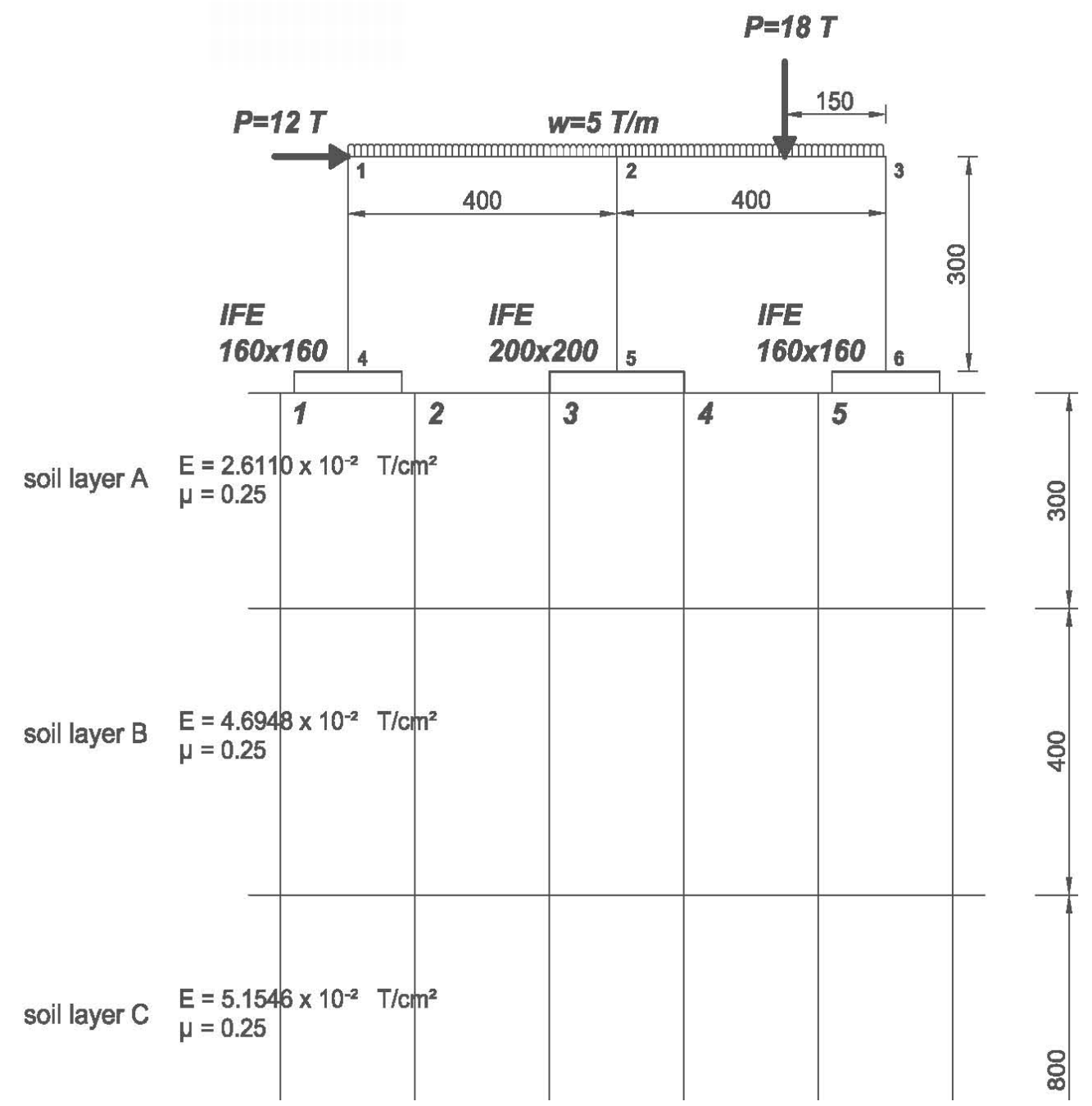

Figure12. 2D frame supported on Isolated Foundation Elements "IFE" (case 3)

Table3. Displacements and reactions calculated in case 3

\begin{tabular}{|c|c|c|c|c|c|c|c|}
\hline & \multicolumn{3}{|c|}{ Displacements } & & \multicolumn{3}{|c|}{ Reactions } \\
\hline & $\mathrm{dx}$ & dy & $\operatorname{Rot} \theta$ & & $\mathrm{Rx}$ & Ry & Mom Z \\
\hline Node & $(\mathrm{cm})$ & $(\mathrm{cm})$ & (rad) & Node & (T) & (T) & $(\mathrm{T}-\mathrm{cm})$ \\
\hline & & & & & & & \\
\hline 1 & 5.031 & -1.664 & -0.01278 & 4 & -1.747 & 7.132 & 158.020 \\
\hline 2 & 5.012 & -5.014 & -0.00656 & 5 & -4.013 & 25.334 & 315.080 \\
\hline 3 & 5.001 & -4.989 & 0.00376 & 6 & -6.239 & 25.534 & 266.250 \\
\hline 4 & 0.000 & -1.648 & -0.01712 & & & & \\
\hline 5 & 0.000 & -4.959 & -0.01850 & & & & \\
\hline 6 & 0.000 & -4.934 & -0.02412 & & & & \\
\hline
\end{tabular}




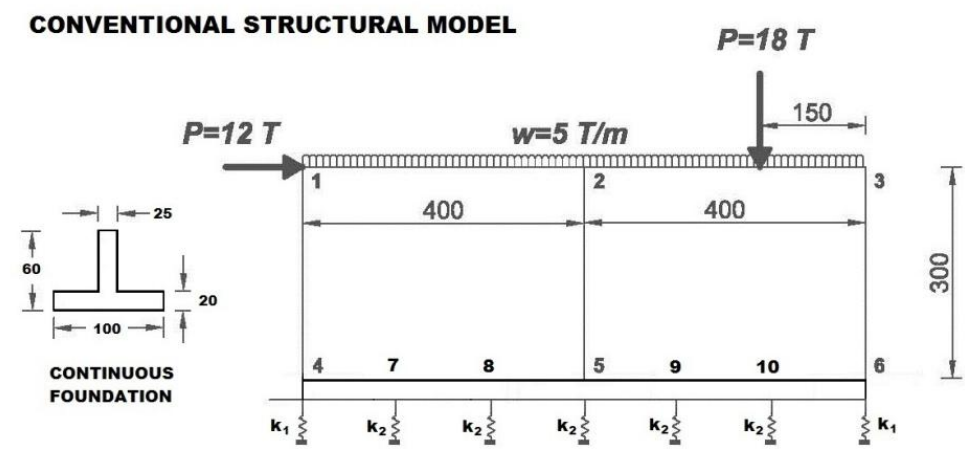

Figure 13. 2D frame supported on classical beams and springs (case 4)

Table4. Displacements and reactions calculated in case 4

\begin{tabular}{|c|c|c|c|c|c|c|c|}
\hline & \multicolumn{3}{|c|}{ Displacements } & & \multicolumn{3}{|c|}{ Reactions } \\
\hline & $\mathrm{dx}$ & dy & $\operatorname{Rot} \theta$ & & $\mathrm{Rx}$ & Ry & Mom Z \\
\hline Node & $(\mathrm{cm})$ & $(\mathrm{cm})$ & (rad) & Node & $(\mathrm{T})$ & $(\mathrm{T})$ & (T-cm) \\
\hline 1 & 1.939 & -0.033 & -0.00419 & 4 & -3.342 & 0.187 & 0.000 \\
\hline 2 & 1.924 & -0.681 & -0.00400 & 5 & -3.706 & 9.742 & 0.000 \\
\hline 3 & 1.915 & -1.467 & 0.00187 & 6 & -4.952 & 11.384 & 0.000 \\
\hline 4 & 0.000 & -0.023 & -0.00178 & 7 & 0.000 & 3.657 & 0.000 \\
\hline 5 & 0.000 & -0.609 & -0.00111 & 8 & 0.000 & 6.760 & 0.000 \\
\hline 6 & 0.000 & -1.423 & -0.00432 & 9 & 0.000 & 11.308 & 0.000 \\
\hline 7 & 0.000 & -0.229 & -0.00142 & 10 & 0.000 & 14.963 & 0.000 \\
\hline 8 & 0.000 & -0.422 & -0.00151 & & & & \\
\hline 9 & 0.000 & -0.707 & -0.00088 & & & & \\
\hline 10 & 0.000 & -0.935 & -0.00273 & & & & \\
\hline
\end{tabular}

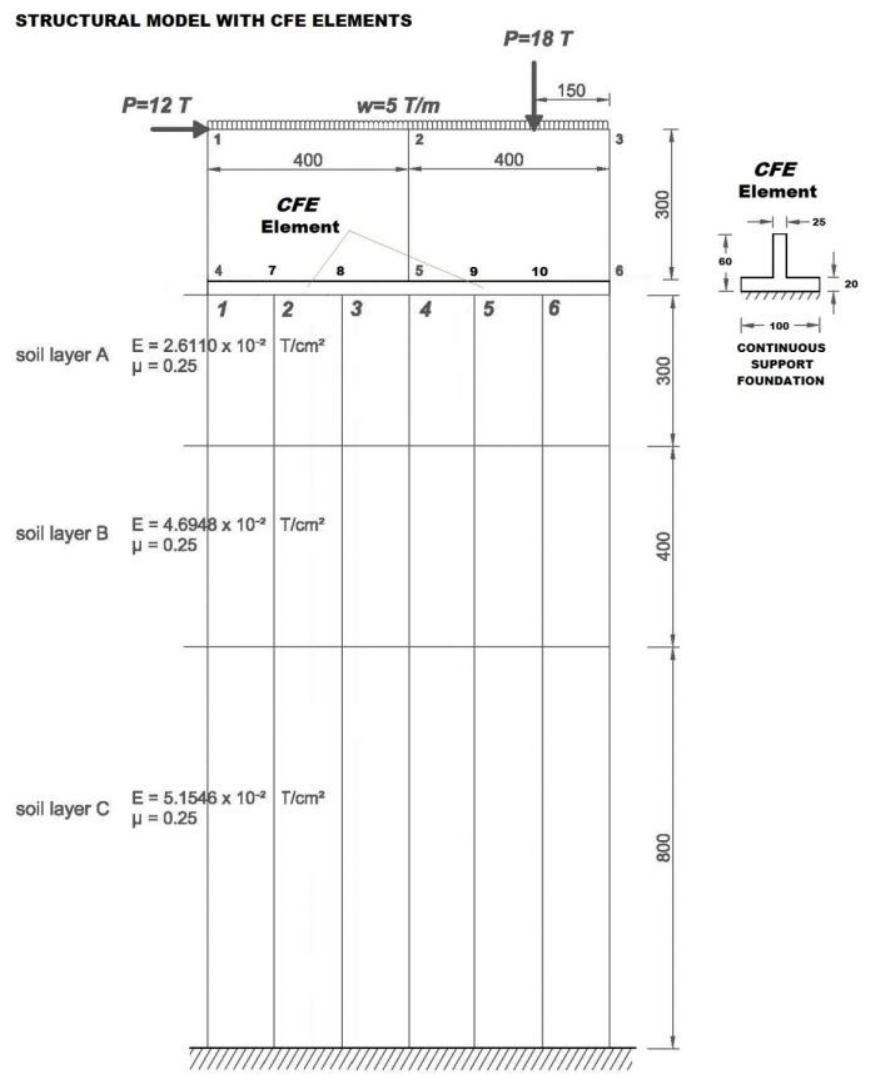

Figure 14. 2D frame supported on a Continuous Foundation Element " $C F E$ " (case 5) 


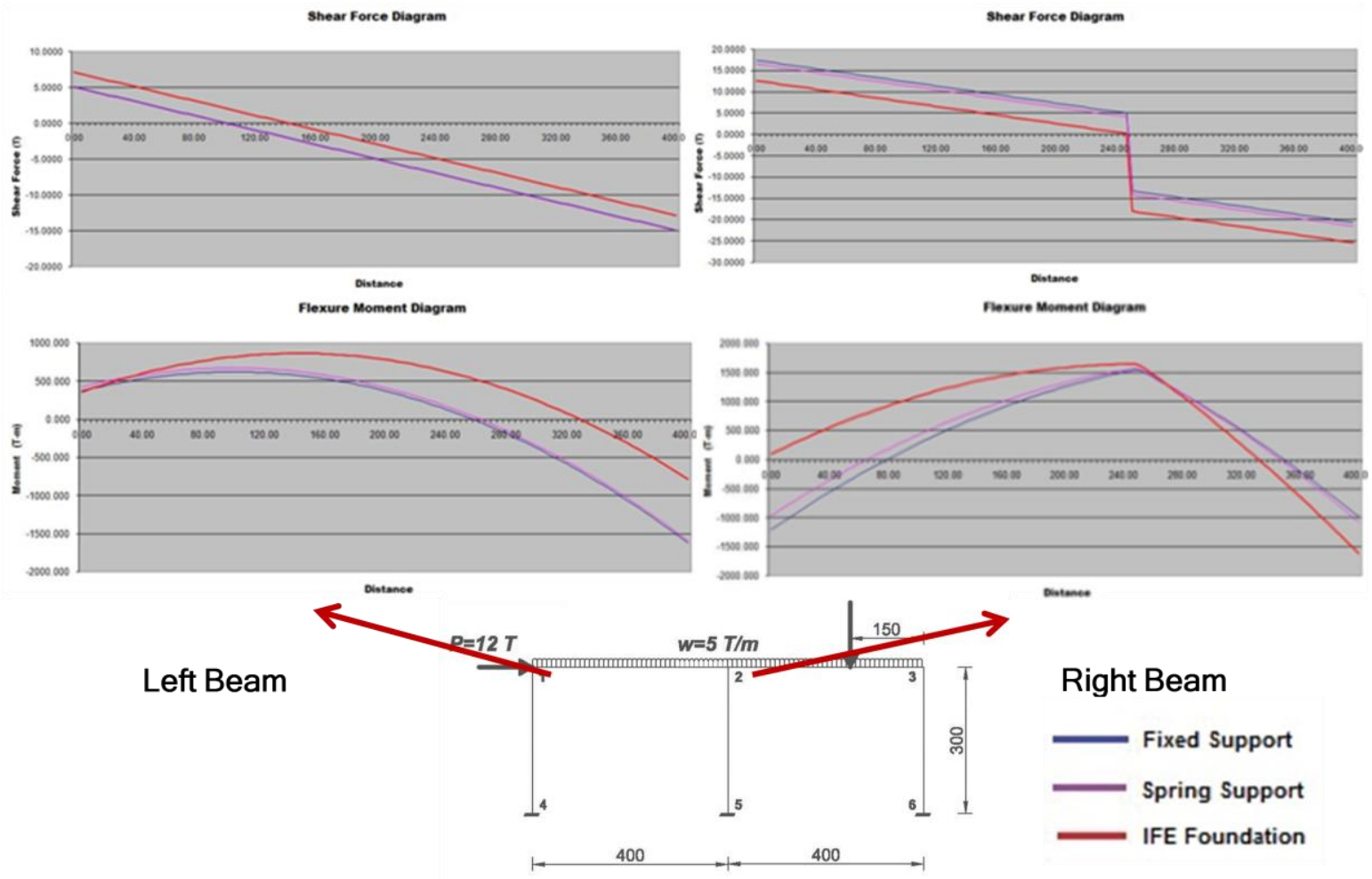

Figure15. Comparison of mechanical forces on upper beams for cases 1, 2, and 3.

\section{CONCLUSIONS}

A simplified numerical strategy for the analysis of the foundation-clay soil system is proposed in this work, in which the properties of the layered soil are taken into account, as well as different deformability's criteria. The strategy is based on the development of two new super-elements called "Isolated Foundation Element (IFE)" and the "Continuous Foundation Element (CFE)". Both super-elements take into account the experimental soil parameters as a part of their internal formulation, which allows calculating the superficial settlements and their effects in the global structural response of the foundation system. This numerical strategy reduce the number of calculations and can give good results about vertical displacements and reaction forces due to its capacity to relate all of the footings and slabs through the use of influence regions. The numerical example shows the importance of including this kind of finite elements in a simple model, affecting the internal distribution of forces in each structural element, which is directly related to the final design of any piece of the whole structural system. 


\section{REFERENCES}

[1] Zeevaert, L. (1973). "Foundation engineering for difficult subsoil conditions", Ed. Van Nostrand Reinhold, New York.

[2] Bowles, J.E. (1997). "Foundation analysis and design”, Ed. McGraw-Hill, New York.

[3] Braja M. Das, (2009), "Shallow Foundations - Bearing Capacity and Settlement", CRC Press

[4] M. Hetenyi, (1946), "Beams on Elastic Foundation", Ed. The University of Michigan Press 